\title{
Perioperative glucocorticoid management based on current evidence
}

\section{Kwon Hui Seo}

Department of Anesthesiology and Pain Medicine, Hallym University Sacred Heart Hospital, Hallym University School of Medicine, Anyang, Korea
Received November 16, 2020 Accepted November 30, 2020

\section{Corresponding author}

Kwon Hui Seo, M.D., Ph.D.

Department of Anesthesiology and

Pain Medicine, Hallym University

Sacred Heart Hospital, Hallym

University School of Medicine, 22

Gwanpyeong-ro 170beon-gil, Dongan-

gu, Anyang 14068, Korea

Tel: 82-31-380-5959

Fax: 82-31-385-3244

E-mail: julianakh@hanmail.net
Glucocorticoid preparations, adreno-cortical steroids, with strong anti-inflammatory and immunosuppressive effects, are widely used for treating various diseases. The number of patients exposed to steroid therapy prior to surgery is increasing. When these patients present for surgery, the anesthesiologist must decide whether to administer perioperative steroid supplementation. Stress-dose glucocorticoid administration is required during the perioperative period because of the possibility of failure of cortisol secretion to cope with the increased cortisol requirement due to surgical stress, adrenal insufficiency, hemodynamic instability, and the possibility of adrenal crisis. Therefore, glucocorticoids should be supplemented at the same level as that of normal physiological response to surgical stress by evaluating the invasiveness of surgery and inhibition of the hypothalamus-pituitary-adrenal axis. Various textbooks and research articles recommend the stress-dose of glucocorticoids during perioperative periods. It has been commonly suggested that glucocorticoids should be administered in an amount equivalent to about $100 \mathrm{mg}$ of cortisol for major surgery because it induces approximately 5 times the normal secretion. However, more studies, with appropriate power, regarding the administration of stress-dose glucocorticoids are still required, and evaluation of patients with possible adrenal insufficiency and appropriate glucocorticoid administration based on surgical stress will help improve the prognosis.

Keywords: Adrenal glands; Adrenal insufficiency; Glucocorticoids; Hypothalmus; Perioperative period; Pituitary gland; Steroids.

\section{INTRODUCTION}

Synthetic glucocorticoids were first introduced in 1949 after the development of a purified preparation, known as cortisone, and became a revolutionary treatment for patients with primary adrenal failure and other acute-chronic inflammatory and autoimmune diseases. In anesthesiology, it is widely used to treat reactive airway diseases, acute nerve injury, nausea or vomiting, inflammatory diseases, and excessive immunosuppression during organ trans- plantation or cardiopulmonary bypass [1]. Shortly after the development of synthetic cortisone, there were two case reports of perioperative secondary adrenal crisis. The two young patients on chronic cortisone therapy stopped steroids before the surgery; they suddenly died after the surgery, and the result of the autopsy showed bilateral adrenal atrophy [2,3]. Since then, it has become common practice to perioperatively administer glucocorticoids at a supra-physiological dose, the so-called stress-dose, to patients on steroid therapy for long duration and in suspected

This is an Open Access article distributed under the terms of the Creative Commons Attribution Non-Commercial License (http://creativecommons.org/licenses/by-nc/4.0) which permits unrestricted non-commercial use, distribution, and reproduction in any medium, provided the original work is properly cited.

Copyright (C) the Korean Society of Anesthesiologists, 2021 
adrenal failure cases.

The number of patients exposed to steroid therapy prior to surgery is increasing as corticosteroids are widely used for systemic administration and as inhalation and topical drugs [4]. Accordingly, clinical trials and retrospective analyses have been conducted regarding stress-dose glucocorticoids administration in the perioperative period for patients on steroid therapy $[5,6]$. However, many clinical trials had a low level of evidence, including unclear criteria for adrenal failure with a limited number of patients, resulting in various proposed recommendations for the administration of glucocorticoids in the perioperative periods [7]. Therefore, this article reviews the physiology of adreno-cortical hormones and indications and applications of stress-dose glucocorticoids in the perioperative periods based on recently published recommendations $[7,8]$.

\section{PHYSIOLOGY OF ADRENOCORTICAL HORMONE SECRETION: HYPOTHALAMUS-PITUITARY-ADRENAL AXIS}

Adrenocorticosteroids are steroid derivatives produced in the adrenal cortex and include three endogenous hormones: glucocorticoid, mineralocorticoid, and androgen. All of them are synthesized when cholesterol is converted to pregnenolone by the cytochrome P450 enzyme [1]. Of these, glucocorticoids are secreted from the zona fasciculata of the adrenal cortex and the most important glucocorticoid is cortisol. Cortisol is an essential hormone for maintaining life. It mediates carbohydrate and protein metabolism, fatty acid transfer, electrolyte and fluid balance, and anti-inflammatory reactions. Cortisol enables the synthesis and release of catecholamines and contributes to normal vascular permeability, vascular tone, and myocardial contraction by regulating $\beta$-receptor synthesis and regulation [1].

The secretion of adrenal cortical hormones is regulated by the hypothalamic-pituitary-adrenal (HPA) axis. The corticotropin-releasing hormone ( $\mathrm{CRH})$ secreted from the hypothalamus stimulates the secretion of adrenocorticotropic hormone (ACTH) in the anterior pituitary gland, and ACTH stimulates the adrenal gland. This positive feedback for cortisol secretion and negative feedback for inhibiting the secretion of CRH and ACTH due to increased cortisol concentration regulates the secretion of cortisol [1].

The secretion of cortisol changes depending on the pulsatile secretion of $\mathrm{CRH}$ and ACTH according to the circadi- an rhythm. Serum cortisol concentration reaches the highest concentration of about $15 \mu \mathrm{g} / \mathrm{dl}$ around 6-9 am, then drops to the lowest concentration of below $2 \mu \mathrm{g} / \mathrm{dl}$ around $11 \mathrm{pm}$ to $1 \mathrm{am}$. The median value of the 24 -h period is approximately $5.2 \mu \mathrm{g} / \mathrm{dl}$ [9]. In normal adults, the adrenal glands produce approximately 5 to $10 \mathrm{mg} / \mathrm{m}^{2} /$ day (body surface area per day) of cortisol, which is equivalent to 5 to $7 \mathrm{mg}$ of oral prednisone or 20 to $30 \mathrm{mg}$ of hydrocortisone $[10,11]$. In plasma, approximately $90 \%$ of circulating cortisol binds to corticosteroid-binding globulin (CBG), an $\alpha_{2}$-globulin binding protein synthesized in the liver [12]. The remaining $5-10 \%$ binds to albumin or circulates freely and exerts an effect on target cells. When plasma cortisol concentration exceeds $20-30 \mu \mathrm{g} / \mathrm{dl}, \mathrm{CBG}$ is saturated, and the concentration of free cortisol increases rapidly [12].

Sudden physiological and mental stress such as trauma, burns, major surgery, hypoglycemia, high fever, low blood pressure, severe exercise, and cold exposure activates the HPA axis and increases blood ACTH and cortisol levels. In a normal response to stress, the blood cortisol concentration increases to $18-20 \mu \mathrm{g} / \mathrm{dl}$ and adrenal cortisol secretion increases up to 30-45 $\mu \mathrm{g} / \mathrm{dl}$ during moderately stressful situations and about $260 \mu \mathrm{g} / \mathrm{dl}$ in a highly stressful life-threatening situation. Increased cortisol levels normalize within approximately 24 to $48 \mathrm{~h}$ after the stress is resolved [11].

\section{ADRENAL INSUFFICIENCY}

Adrenal insufficiency (AI) is the inability of the adrenal glands to produce adequate amounts of corticosteroids in response to various pathophysiologic states; this condition can be classified into primary, secondary, and tertiary AI depending on the cause [11]. Primary AI is an abnormality in the adrenal gland itself and is caused by the destruction of the adrenal cortex due to autoimmune diseases, viral and tuberculosis infection, hemorrhage, metastatic cancer, and sepsis. Secondary AI is rare but is caused by impaired production of ACTH or CRH due to damage or dysfunction caused by diseases of the pituitary gland or hypothalamus. Tertiary AI is the most common form, widely included in secondary AI, and is typically caused by inhibition of the hypothalamus or pituitary due to iatrogenic corticosteroid therapy; the degree of adrenal dysfunction is variable and sometimes reversible. In these patients, mineralocorticoid secretion is not affected and only cortisol production is reversibly inhibited [13]. Tertiary AI rarely occurs when oral prednisone dosage is less than $5 \mathrm{mg}$ or when steroid is tak- 
en for a short period of less than 2 weeks, regardless of the dosage. However, AI has been reported to occur in more than $60 \%$ of patients taking high-dose oral prednisone (> $40 \mathrm{mg}$ ) even after only about 6 days $[11,14]$.

\section{Clinical signs of adrenal crisis}

Patients with long-term steroid administration or severe illness have a reduced cortisol response to stress, which causes risk of an acute adrenal crisis. Symptoms of acute adrenal crisis in awake patients are presented in Table 1 [15]. In patients under anesthesia, hypotension, which does not respond to fluid administration, has been considered the most important sign of perioperative adrenal crisis [7]. Symptoms and signs that occur earlier than hypotension include non-specific changes in consciousness and cognitive decline and persistent fever [8]. Laboratory examinations may show hypoglycemia, hyponatremia, and hyperkalemia. Since most of these symptoms are non-specific, it is necessary to exclude causes other than AI. However, since adrenal crisis is a life-threatening condition, it should be immediately recognized and corrected by the administration of stress-doses of steroids, fluids, and vasopressors [8].

\section{SURGERY INDUCED CORTISOL STRESS RESPONSE}

Surgery causes a stress response with a wide range of endocrine, immune, and cardiovascular effects. During major surgery, proinflammatory cytokines, $\mathrm{CRH}, \mathrm{ACTH}$, and cortisol levels increase proportionally, resulting in an increase in cortisol secretion up to approximately 5-10 times the normal secretion, that is, $75-150 \mathrm{mg} /$ day $[16,17]$. After surgery, the diurnal secretion of cortisol malfunctions temporarily, and the serum concentration of cortisol rises due to

Table 1. Signs of Adrenal Crisis [15]

Signs of adrenal crisis

Dehydration, hypotension

Nausea and vomiting with a history of weight loss and anorexia

Abdominal pain (“acute abdomen”)

Unexplained hypoglycemia

Unexplained fever

Hyponatremia, hyperkalemia, azotemia, hypercalcemia, eosinophilia Hyperpigmentation or vitiligo

Other autoimmune endocrine deficiencies (hypothyroidism or gonadal failure) surgical stress [11].

In a recent meta-analysis, the change in serum cortisol concentration before and after surgery in patients without steroid therapy was analyzed in 71 studies since the 1990s [18]. In this study, the invasiveness of surgery was divided into three stages from grade 1 to 3 according to the modified Johns Hopkins surgical criteria [19]. Minor to moderately invasive procedures with less bleeding (potential blood loss < $500 \mathrm{ml}$ ) were included in grade 1; for example, breast biopsy, removal of minor skin or subcutaneous lesions, myringotomy tubes, hysteroscopy, cystoscopy, vasectomy, circumcision, fiberoptic bronchoscopy, diagnostic laparoscopy, dilatation, and curettage. Moderately to significantly invasive procedures (potential blood loss 500 $1,500 \mathrm{ml}$ ) were included in grade 2 ; for example, thyroidectomy, hysterectomy, myomectomy, cystectomy, cholecystectomy, laminectomy, hip/knee replacement, nephrectomy, and major laparoscopic procedures. Highly invasive procedures (potential blood loss $>1,500 \mathrm{ml}$ ) were included in Grade 3, for example, major reconstruction of the gastrointestinal tract, major genitourinary surgery, cardiothoracic procedures, and intracranial procedures. In this review, it was found that the grade of surgery significantly affected cortisol secretion [18]. Patients undergoing grade 1 surgery did not show an intraoperative cortisol peak, and postsurgical cortisol concentrations were similar to those at baseline. Nevertheless, when compared to published data on healthy, unstressed adults, the mean cortisol output over the first $24 \mathrm{~h}$ after grade I surgical procedure was approximately doubled. Patients undergoing grade 2 and 3 surgery had 3.5-4 times higher cortisol output than that of healthy, unstressed individuals within the first 24 -h postoperative period. Moreover, in both grade 2 and 3 surgeries, mean cortisol values remained elevated in comparison with the baseline measurements up to postoperative day 7 .

Due to ethical issues, only a few studies have investigated the change in cortisol concentration and the incidence of AI after discontinuation of steroids in patients taking steroids. In 1973, Kehlet and Binder [16] investigated the occurrence of acute AI after steroid discontinuation in 73 patients undergoing major surgery, including splenectomy and colon resection. Patients took 5-80 mg of prednisone for various periods in this study. As a result, about $10 \%$ of patients developed perioperative hypotension, but only 3 patients showed low blood cortisol levels, and most of them were treated by fluid administration. They also measured cortisol concentration in patients undergoing major 
abdominal surgery and minor procedures such as hand surgery or uterine curettage, and estimated cortisol secretion due to surgical stress was found to be $75-150 \mathrm{mg} /$ day and $50 \mathrm{mg} /$ day for major and minor surgeries, respectively. The results of this study formed the rationale for subsequent recommendations of perioperative glucocorticoid supplementation $[7,20]$.

De Lange and Kars [5] and Khazen and El-Hussuna [6] investigated the incidence of AI following administration of stress-doses or conventional maintenance doses of glucocorticoids based on prospective and retrospective studies. The randomized controlled trials included in their meta-analysis mostly targeted minor to moderate surgery with a limited number of patients, and both meta-analyses concluded that the incidence of perioperative AI was very low $[5,6]$. These studies reported that patients taking less than 5-10 mg/day of prednisone did not have adverse side effect of AI, even if only the daily dose was maintained during relatively less invasive surgery [21-23].

\section{PHYSIOLOGICAL RATIONALE FOR PERIOPERATIVE GLUCOCORTICOID SUPPLEMENTATION}

Despite the low incidence of surgery-induced AI, several major pathophysiologic mechanisms support the necessity of glucocorticoid administration in the perioperative period.

\section{Vascular tone and maintenance of blood pressure}

Glucocorticoids have a permissive effect on vascular tone and maintenance of blood pressure [24]. Glucocorticoids alone do not increase blood pressure, but when administered with a vasopressor, glucocorticoids enhance vascular reactivity to vasopressors. The effect of glucocorticoid on vascular tone is exerted by inhibition of the synthesis of prostacyclin $\mathrm{I}_{2}\left(\mathrm{PGI}_{2}\right)$, a potent vasodilator, in the vascular endothelium [25]. If the inhibitory effect on vascular tone disappears due to a decrease in cortisol response, it may lead to increased production of $\mathrm{PGI}_{2}$, vasodilation, and hypotension.

\section{Catecholamine synthesis and secretion}

Cortisol is involved in catecholamine synthesis and mediates the release of catecholamine from sympathetic nerve cells by directly regulating the activity of phenylethanolamine N-methyltransferase, an enzyme that catalyzes the conversion of norepinephrine to epinephrine in the adrenal medulla [26]. Cortisol also mediates catecholamine release from sympathetic cells [27].

\section{Myocardial contractility}

Cortisol helps the myocardium adapt to perioperative stress [28]. In animal studies, acute adrenal failure caused reduced myocardial contractility due to a decrease in the activity of myofibrillar adenosine triphosphatase, which is directly dependent on glucocorticoids [29]. In patients with hemodynamically unstable secondary AI, bolus intravenous hydrocortisone increases the stroke work index of the left ventricle [30].

\section{SYNTHETIC ADRENOCORTICOIDS}

All synthetic glucocorticoids are derivatives of cortisol, an endogenous glucocorticoid. Drugs used as therapeutic glucocorticoids include hydrocortisone, prednisolone, and dexamethasone (Table 2) [1]. Among these drugs, hydrocortisone, which has the same structure as cortisol, is the most commonly used synthetic glucocorticoid. Prednisone is an inactive prodrug that is activated to prednisolone by $11 \beta$-hydroxysteroid dehydrogenase after administration [31]. Synthetic corticosteroids have different glucocorticoid and mineralocorticoid activities. Table 2 shows the relative efficacy of commonly used corticosteroids compared to

Table 2. Relative Potency of Synthetic Steroids [1]

\begin{tabular}{|c|c|c|c|c|}
\hline Drugs & Glucocorticoid activity & Mineralocorticoid activity & Equivalent dose (mg) & Duration of action (h) \\
\hline Hydrocortisone (cortisol) & 1 & 1 & 20 & $8-12$ \\
\hline Prednisone & 4 & 0.8 & 5 & $12-36$ \\
\hline Prednisolone & 4 & 0.8 & 5 & $12-36$ \\
\hline Methylprednisolone & 5 & 0.5 & 4 & $12-36$ \\
\hline Dexamethasone & $30-40$ & 0 & $0.5-0.75$ & $36-54$ \\
\hline Fludrocortisone & 10 & 250 & 2 & 24 \\
\hline Aldosterone & 0 & 3000 & & \\
\hline
\end{tabular}


that of hydrocortisone [1]. When adrenal gland function is reduced, corticosteroids are used to replace both glucocorticoids and mineralocorticoids. Therefore, in patients with primary AI in which mineralocorticoids are not secreted, dexamethasone is not appropriate and synthetic mineralocorticoids, fludrocortisone, and hydrocortisone should be administered [7]. Secondary and tertiary AI are caused by a lack of glucocorticoids, so the administration of drugs with mineralocorticoid activity may cause side effects such as dose-dependent edema, fluid retention, and hypokalemia [7]. Therefore, when a high dose of hydrocortisone of 100 mg or more is required in patients with secondary and tertiary AI, switching to a drug with a higher glucocorticoid activity ratio than that of mineralocorticoid such as methylprednisolone should be considered [24].

\section{Side effects of prolonged administration of high dose of glucocorticoid}

Continuous administration of high doses of glucocorticoids after surgery can cause unwanted side effects [31]. Glucocorticoids promote gluconeogenesis in the liver and proteolysis and adipolysis in muscles, resulting in hyperglycemia. In addition, continuous glucocorticoid administration results in sodium retention, subsequent plasma volume increase, and intensifies vasopressor response to angiotensin II and catecholamines, leading to hypertension [32]. Glucocorticoids inhibit cytokine signaling and the synthesis of matrix metalloproteinases and collagen, which play an important role in wound healing [33]. In addition, it can cause gastrointestinal bleeding and various psychiatric symptoms [7]. However, most side effects occur in proportion to the duration of glucocorticoid administration, therefore, the incidence of side effects after shortterm treatment is low even with high doses.

\section{PERIOPERATIVE STRESS-DOSE GLUCOCORTICOID}

Glucocorticoids should be administered during the perioperative period because cortisol secretion in response to surgical stress may fail, resulting in AI, hemodynamic instability, and adrenal crisis. Therefore, the dose of glucocorticoid should be administered at the same level as that of normal physiological response to the surgical stress after evaluating the invasiveness of surgery and inhibition of the HPA axis [24]. If there is no suppression of the HPA axis or the requirement due to surgical stress does not exceed the maintenance dose of glucocorticoids already being taken, a perioperative stress-dose of glucocorticoid is not required unless the patient shows signs of AI [7]. However, when glucocorticoid requirement increases rapidly due to surgical stress, and the inhibition of the HPA axis is clinically important, the administration of stress doses should be considered $[7,8]$.

\section{Approach according to HPA axis suppression}

\section{Nonsuppressed HPA axis}

Steroid dose and duration affect HPA axis suppression. Regardless of duration, the risk of HPA axis suppression is low if the prednisone dose taken in the morning does not exceed $5 \mathrm{mg} /$ day ( $\approx$ methylprednisolone $4 \mathrm{mg} /$ day, dexamethasone $0.5 \mathrm{mg} /$ day, hydrocortisone $20 \mathrm{mg} /$ day) or 10 mg of prednisone every other day. In addition, if any dose of glucocorticoid is administered for less than three weeks, the HPA axis is less likely to be suppressed. These patients do not require additional administration of glucocorticoids or tests to assess the HPA axis [7].

\section{Patients with suppressed HPA axis}

Patients on daily dose of prednisone exceeding $20 \mathrm{mg}$ for a period of more than three weeks and patients with symptoms of Cushing syndrome who are taking glucocorticoids are at high risk of HPA axis suppression [7]. These patients should be administered perioperative supplemental glucocorticoids according to the invasiveness of surgery [7].

\section{Unknown HPA axis suppression}

Besides these patients, patients taking prednisone at 5-20 mg/day or equivalent doses over a period of three or more weeks may experience various ranges of HPA axis suppression depending on their age, and dosage and duration of administration [34]. Even in cases with discontinued exposure of steroids, patients who inhaled high-dose steroids or high-potency topical steroids should be tested for adrenal function preoperatively and supplemental glucocorticoids should be administered based on the results of the test. There is a risk of HPA axis inhibition when inhaled glucocorticoid fluticasone $\geq 750 \mu \mathrm{g} /$ day (or beclomethasone $\geq 1,500 \mu \mathrm{g} /$ day $\approx$ prednisolone $\geq 10 \mathrm{mg} /$ day) is administered for more than 3 weeks before surgery $[35,36]$. The absorption rate of topical steroids varies depending on the period of use, strength, and application site, but when 
topical steroids with high potency are used for $>2 \mathrm{~g} /$ day for more than 2 weeks, suppression of the HPA axis may occur [37]. In addition, patients who have received three or more intra-articular or spinal glucocorticoid injections within three months prior to surgery, have symptoms of AI, or Cushing's syndrome, the HPA axis needs to be evaluated [38].

\section{Assessment of HPA axis suppression}

\section{Morning serum cortisol}

Measurement of morning serum cortisol concentrations before $8 \mathrm{am}$ after stopping glucocorticoids for $24 \mathrm{~h}$ is a good screening test for assessing secondary and tertiary AI symptoms [39]. If the morning serum cortisol concentration is lower than $5 \mu \mathrm{g} / \mathrm{dl}$, suppression of the HPA axis may be suspected and the administration of additional glucocorticoids is required [7]. If the morning cortisol concentration is greater than $10 \mu \mathrm{g} / \mathrm{dl}$, it can be considered that there is no inhibition of the HPA axis, and the usual dose of glucocorticoid is taken until the day of surgery and no additional administration is required [7]. If the morning cortisol concentration is 5 to $10 \mu \mathrm{g} / \mathrm{dl}$, ACTH stimulation tests are conducted, or glucocorticoids are administered based on experience.

\section{Short ACTH stimulation tests}

The ACTH stimulation test determines whether adrenal function is inhibited by administering synthetic ACTH (cosyntropin $250 \mu \mathrm{g}$ ); the concentration of serum cortisol are measured $30 \mathrm{~min}$ after the administration of ACTH [24]. If cortisol concentration is higher than $18 \mu \mathrm{g} / \mathrm{dl}$, it can be determined that proper adrenal function is maintained and additional administration of glucocorticoid is not required [7].

\section{RECOMMENDED DOSE OF GLUCOCORTICOID ACCORDING TO SURGICAL STRESS}

Based on recent studies, recommendations were published in Anesthesiology in 2017 and Anaesthesia in 2020 $[7,8]$. As presented in Table 3 , Liu et al. proposed recommendations based on estimated daily cortisol secretions according to the invasiveness of surgery $[7,16,17]$.

Recently, the Royal College of Anaesthetists and the Endocrinology Society of the United Kingdom also published guidelines in Anaesthesia [8], but they are different from those of Liu et al. [7]. They have similarity in that they recommend administering stress-dose steroids to patients with primary and secondary AI and HPA axis suppression. However, they recommended $100 \mathrm{mg}$ of hydrocortisone for all patients undergoing minor procedures as well as major surgeries [8]. After the publication of this recommendation, when questions arose about administering the same dose for a relatively simple operation [40], the authors responded that this dose may not be appropriate for simple proce-

Table 3. Surgical Stress according to Procedures and Recommended Dosing of Glucocorticoid [7]

\begin{tabular}{|c|c|c|c|}
\hline Type of surgery & Estimated cortisol secretion rate & Examples & Recommended dosing \\
\hline \multirow[t]{2}{*}{ Superficial } & 8-10 mg/day & Dental surgery & Usual daily dose \\
\hline & & Biopsy & \\
\hline \multirow[t]{4}{*}{ Minor } & 50 mg/day & Inguinal hernia repair & Usual daily dose plus \\
\hline & & Colonoscopy & \\
\hline & & Uterine curettage & Hydrocortisone 50 mg IV before incision \\
\hline & & Hand surgery & Hydrocortisone $25 \mathrm{mg}$ IV every $8 \mathrm{~h} \times 24 \mathrm{~h}$ \\
\hline \multirow[t]{5}{*}{ Moderate } & $75-150 \mathrm{mg} /$ day & Lower extremity revascularization & Then usual daily dose \\
\hline & & Total joint replacement & \\
\hline & & Cholecystectomy & \\
\hline & & Colon resection & \\
\hline & & Abdominal hysterectomy & \\
\hline \multirow[t]{6}{*}{ Major } & 75-150 mg/day & Esophagectomy & Usual daily dose plus \\
\hline & & Total proctocolectomy & Hydrocortisone 100 mg IV before incision \\
\hline & & Major cardiac/vascular surgery & Followed by continuous IV hydrocortisone $200 \mathrm{mg}$ (> $24 \mathrm{~h}$ ) \\
\hline & & Hepaticojejunostomy & or \\
\hline & & Delivery & Hydrocortisone $50 \mathrm{mg}$ IV every $8 \mathrm{~h} \times 24 \mathrm{~h}$ \\
\hline & & Trauma & Taper dose by half per day until usual daily dose reached \\
\hline
\end{tabular}

IV: intravenously. 
dures [41]. Therefore, we can only refer to their recommendations for major surgery. According to this guideline, for patients undergoing major surgery, hydrocortisone $100 \mathrm{mg}$ or dexamethasone 6-8 $\mathrm{mg}$ should be administered at time of induction of anesthesia, followed by immediate initiation of a continuous infusion of hydrocortisone $200 \mathrm{mg} /$ day until the patients can be administered double the pre-surgical oral dose [8].

\section{CONCLUSION}

Cortisol has a variety of critical physiological actions, and an increase in concentration due to surgical stress is important for maintaining hemodynamic stability during surgery. Although studies with more appropriate evidence are still required, evaluation of patients with possible AI and glucocorticoid administration according to surgical stress is crucial and can improve prognosis.

\section{CONFLICTS OF INTEREST}

No potential conflict of interest relevant to this article was reported.

\section{ORCID}

Kwon Hui Seo, https://orcid.org/0000-0003-4397-9207

\section{REFERENCES}

1. Keegan MT. Endocrine Pharmacology. In: Pharmacology and physiology for anesthesia: foundations and clinical application. 2nd ed. Edited by Hemmings HC Jr, Egan TD: Philadelphia, Elsevier. 2019, pp 708-31.

2. Fraser CG, Preuss FS, Bigford WD. Adrenal atrophy and irreversible shock associated with cortisone therapy. J Am Med Assoc 1952; 149: 1542-3.

3. Lewis L, Robinson RF, Yee J, Hacker LA, Eisen G. Fatal adrenal cortical insufficiency precipitated by surgery during prolonged continuous cortisone treatment. Ann Intern Med 1953; 39: $116-26$.

4. Marik PE, Varon J. Requirement of perioperative stress doses of corticosteroids: a systematic review of the literature. Arch Surg 2008; 143: 1222-6

5. de Lange DW, Kars M. Perioperative glucocorticosteroid supplementation is not supported by evidence. Eur J Intern Med 2008; 19: 461-7.
6. Khazen BF, El-Hussuna A. The use of a perioperative supra-physiological dose of glucocorticoid is not supported by evidence - a systematic review. Dan Med J 2018; 65: A5488.

7. Liu MM, Reidy AB, Saatee S, Collard CD. Perioperative steroid management: approaches based on current evidence. Anesthesiology 2017; 127: 166-72.

8. Woodcock T, Barker P, Daniel S, Fletcher S, Wass JAH, Tomlinson JW, et al. Guidelines for the management of glucocorticoids during the peri-operative period for patients with adrenal insufficiency: guidelines from the Association of Anaesthetists, the Royal College of Physicians and the Society for Endocrinology UK. Anaesthesia 2020; 75: 654-63.

9. Debono M, Ghobadi C, Rostami-Hodjegan A, Huatan H, Campbell MJ, Newell-Price J, et al. Modified-release hydrocortisone to provide circadian cortisol profiles. J Clin Endocrinol Metab 2009; 94: 1548-54.

10. Esteban NV, Loughlin T, Yergey AL, Zawadzki JK, Booth JD, Winterer JC, et al. Daily cortisol production rate in man determined by stable isotope dilution/mass spectrometry. J Clin Endocrinol Metab 1991; 72: 39-45.

11. Coursin DB, Wood KE. Corticosteroid supplementation for adrenal insufficiency. JAMA 2002; 287: 236-40.

12. Chrousos GP. Chapter 39: adrenocorticosteroids \& adrenocortical antagonists. In: Basic \& clinical pharmacology. 14th ed. Edited by Katzung BG: New York, McGraw-Hill. 2018.

13. MacKenzie CR, Goodman SM. Stress dose steroids: myths and perioperative medicine. Curr Rheumatol Rep 2016; 18: 47.

14. Woods CP, Argese N, Chapman M, Boot C, Webster R, Dabhi V, et al. Adrenal suppression in patients taking inhaled glucocorticoids is highly prevalent and management can be guided by morning cortisol. Eur J Endocrinol 2015; 173: 633-42.

15. Garcia JEL, Hill GE, Joshi GP. Perioperative stress dose steroids: is it really necessary? ASA Newsl 2013; 77: 32-5.

16. Kehlet H, Binder C. Adrenocortical function and clinical course during and after surgery in unsupplemented glucocorticoid-treated patients. Br J Anaesth 1973; 45: 1043-8.

17. Salem M, Tainsh RE Jr, Bromberg J, Loriaux DL, Chernow B. Perioperative glucocorticoid coverage. A reassessment 42 years after emergence of a problem. Ann Surg 1994; 219: 416-25.

18. Prete A, Yan Q, Al-Tarrah K, Akturk HK, Prokop LJ, Alahdab F, et al. The cortisol stress response induced by surgery: a systematic review and meta-analysis. Clin Endocrinol (Oxf) 2018; 89: 554-67.

19. Donati A, Ruzzi M, Adrario E, Pelaia P, Coluzzi F, Gabbanelli V, et al. A new and feasible model for predicting operative risk. Br J Anaesth 2004; 93: 393-9.

20. Lamberts SW, Bruining HA, de Jong FH. Corticosteroid therapy 
in severe illness. N Engl J Med 1997; 337: 1285-92.

21. Glowniak JV, Loriaux DL. A double-blind study of perioperative steroid requirements in secondary adrenal insufficiency. Surgery 1997; 121: 123-9.

22. Thomason JM, Girdler NM, Kendall-Taylor P, Wastell H, Weddel A, Seymour RA. An investigation into the need for supplementary steroids in organ transplant patients undergoing gingival surgery. A double-blind, split-mouth, cross-over study. J Clin Periodontol 1999; 26: 577-82.

23. Zaghiyan K, Melmed GY, Berel D, Ovsepyan G, Murrell Z, Fleshner P. A prospective, randomized, noninferiority trial of steroid dosing after major colorectal surgery. Ann Surg 2014; 259: $32-7$.

24. Axelrod L. Perioperative management of patients treated with glucocorticoids. Endocrinol Metab Clin North Am 2003; 32: 367-83.

25. Axelrod L. Inhibition of prostacyclin production mediates permissive effect of glucocorticoids on vascular tone. Perturbations of this mechanism contribute to pathogenesis of Cushing's syndrome and Addison's disease. Lancet 1983; 1: 904-6.

26. Wong DL, Siddall B, Wang W. Hormonal control of rat adrenal phenylethanolamine N-methyltransferase. Enzyme activity, the final critical pathway. Neuropsychopharmacology 1995; 13: 223-34.

27. Shi LJ, He HY, Liu LA, Wang CA. Rapid nongenomic effect of corticosterone on neuronal nicotinic acetylcholine receptor in PC12 cells. Arch Biochem Biophys 2001; 394: 145-50.

28. Sabourdin N. Steroids: the evidence. The rationale for perioperative glucocorticoid supplementation for patients under chronic steroid treatment. Curr Anesthesiol Rep 2015; 5: 140-6.

29. Bhaskar M, Stith RD, Brackett DJ, Wilson MF, Lerner MR, Reddy YS. Changes in myocardial contractile protein ATPases in chronically adrenalectomized rats with and without glucocorticoid replacement. Biochem Med Metab Biol 1989; 42: 118-24.

30. Bouachour G, Tirot P, Varache N, Gouello JP, Harry P, Alquier P.
Hemodynamic changes in acute adrenal insufficiency. Intensive Care Med 1994; 20: 138-41.

31. Czock D, Keller F, Rasche FM, Häussler U. Pharmacokinetics and pharmacodynamics of systemically administered glucocorticoids. Clin Pharmacokinet 2005; 44: 61-98.

32. Rhen T, Cidlowski JA. Antiinflammatory action of glucocorticoids--new mechanisms for old drugs. N Engl J Med 2005; 353: 1711-23.

33. Sato S, Kim T, Arai T, Maruyama S, Tajima M, Utsumi N. Comparison between the effects of dexamethasone and indomethacin on bone wound healing. Jpn J Pharmacol 1986; 42: 71-8.

34. Freudzon L. Perioperative steroid therapy: where's the evidence? Curr Opin Anaesthesiol 2018; 31: 39-42.

35. Todd GR, Acerini CL, Ross-Russell R, Zahra S, Warner JT, McCance D. Survey of adrenal crisis associated with inhaled corticosteroids in the United Kingdom. Arch Dis Child 2002; 87: 457-61.

36. Lipworth BJ. Systemic adverse effects of inhaled corticosteroid therapy: a systematic review and meta-analysis. Arch Intern Med 1999; 159: 941-55.

37. Hengge UR, Ruzicka T, Schwartz RA, Cork MJ. Adverse effects of topical glucocorticosteroids. J Am Acad Dermatol 2006; 54: $1-15$.

38. Lansang MC, Farmer T, Kennedy L. Diagnosing the unrecognized systemic absorption of intra-articular and epidural steroid injections. Endocr Pract 2009; 15: 225-8.

39. Hägg E, Asplund K, Lithner F. Value of basal plasma cortisol assays in the assessment of pituitary-adrenal insufficiency. Clin Endocrinol (Oxf) 1987; 26: 221-6.

40. McConachie I. Peri-operative glucocorticoid guidelines. Anaesthesia 2020; 75: 1394.

41. Vercueil A; Working Party. Guidelines for the management of glucocorticoids during the peri-operative period for patients with adrenal insufficiency: a reply. Anaesthesia 2020; 75: 13989. 